\title{
Performance Analysis of a THz Proximity Wireless Communication Based on RTD
}

\author{
Rafael Nobrega ${ }^{1,4}$ and Ulysses Duarte ${ }^{1}$ \\ Academic Area of Electrical Engineering \\ Federal Institute of Minas Gerais ${ }^{1}$ \\ Formiga, Brazil \\ \{rafael.nobrega, ulysses.rondina\}@ifmg.edu.br
}

\author{
Ivan Glesk ${ }^{3}$ \\ Faculty of Engineering \\ University of Strathclyde ${ }^{3}$ \\ Glasgow, United Kingdom \\ ivan.glesk@strath.ac.uk
}

\author{
Thiago Raddo ${ }^{2}$ \\ Institute for Photonic Integration \\ Eindhoven University of Technology ${ }^{2}$ \\ Eindhoven, The Netherlands \\ t.r.raddo@tue.nl
}

\author{
Anderson Sanches ${ }^{3,4}$ and Murilo Loiola ${ }^{4}$ \\ Engineering, Modeling, and Applied Social Sciences Center \\ Federal University of $\mathrm{ABC}^{4}$ \\ Santo André, Brazil \\ \{anderson.sanches, murilo.loiola\}@ufabc.edu.br
}

\begin{abstract}
This paper presents a comprehensive analysis of THz proximity wireless communication comprising a resonant tunneling diode (RTD) as a solid-state signal source. For such a system, it is investigated how the RTD-based oscillator performs under direct current (DC) bias applied within the negative differential region (NDR) to provide oscillations in the $\mathbf{T H z}$ range. Specifically, it is accounted for the DC bias effect over the reactive elements that composed the small signal model of the oscillator composed by RTD device and bowtie antenna. The mathematical formalism developed here does require the use of complicated methods to assess the current versus voltage (I-V) characteristics of the RTD-based oscillators, which makes the performance analysis straightforward. The results indicated that the RTD-based oscillator could stabilize very highfrequency oscillations and the $\mathrm{THz}$ proximity link considered can perform error-free transmissions at the short-range link up to $27 \mathrm{~m}$.
\end{abstract}

\section{Keywords-Wireless communication, THz frequency, RTD, proximity link, semi-analytical approach}

\section{INTRODUCTION}

The wide bandwidth available in the terahertz $(\mathrm{THz})$ frequency range $(100 \mathrm{GHz}-10 \mathrm{THz})$ has attracted much attention by allowing the deployment of high capacity wireless communications systems [1]-[3]. As such THz range is located between lightwaves and millimeter waves, both optical- and electron-based devices have been investigated as $\mathrm{THz}$ sources. In addition, it is well known that compact and coherent solid-state sources capable of high-speed modulation are mandatory to enable these applications [4]. For commercial applications, it is also desirable that these sources be highly efficient, i.e., able to transmits relatively high output power at significant low direct current (DC) consumption. Fortunately, all these requirements can be fully satisfied by one of the most prominent electronic devices available, namely resonant tunneling diode (RTD). Oscillators based on RTD is known to have the highest maximum oscillation frequency at room temperature of the inherent negative differential conductance (NDC) due to the resonant tunneling among the electronic devices [4], [5].

Although RTD-based systems have been demonstrated in the THz frequency range [1], [4], the theoretical response of the device in the employing circuits are not thoroughly investigated. Over the years, a great deal of effort has been dedicated to the development of efficient approaches to modeling oscillators based on RTDs [2], [3], [6], [7]. Nonetheless, whereas there is a lack of modeling for the behavior of such circuits operating at $\mathrm{THz}$ frequencies, the

This work was supported by grant from São Paulo Research Foundation (FAPESP) (grant number 2017/26338-9) approaches available in the literature leads to enormous complications to solve its mathematical formalisms and then infer the oscillators' parameters. Based on the earlier discussion, one might see that there are still some aspects of RTD-based circuits modeling that are yet to be accounted for. For example, a model that adequately includes not only the RTD reactive elements dependence over the frequency of operation at $\mathrm{THz}$ range but also have a straightforward solution.

In this context, this paper presents a comprehensive analysis of an RTD-based oscillator and its application at a $\mathrm{THz}$ proximity wireless communication system. For this, it is derived a new expression to evaluate the width of the depletion region and investigated how the DC voltage performs on the oscillation frequency in order to achieve the generation of $\mathrm{THz}$ waves.

\section{MATHEMATICAL FRAMEWORK}

In this section, it is developed the mathematical formalism that will be utilized to model the $\mathrm{THz}$ proximity wireless communication system. Such a system is based on an oscillator circuit and passive structures characterized by a hemispherical lens and an antenna, as shown in Fig. 1.

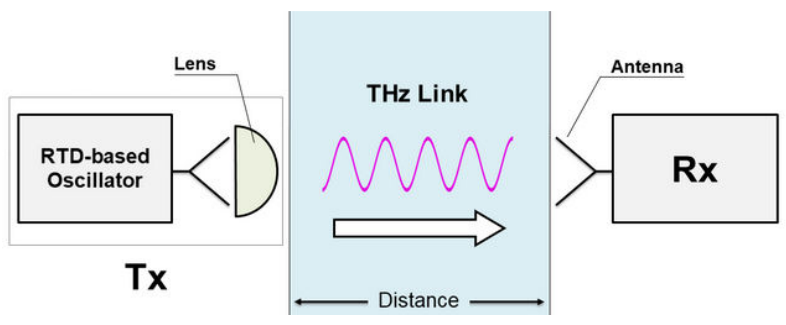

Figure 1. Block diagram of the THz proximity wireless communication system based on RTD.

At the transmitter side, the oscillator circuit consists of an RTD device connected for an antenna. Initially, the oscillation occurs when the intrinsic NDC of the RTD compensates for both the resonator and antenna losses. Here, it is adopted an RTD approach which comprises a parallel association between the conductance $G_{r t d}$ caused by double barrier tunneling and the overall capacitance $C_{r t d}$ of the device. This capacitance is related to the parallel plate, the space-charge accumulation, accumulation and depletion regions adjacent to the barriers, and the charge storage in the quantum well. Also, the modeling considers the previous association in tandem with the series contact and bulk resistance $R_{S}$ and the inductance established from the bond wires in the packaged diode [1]. The antenna, by its turn, can equivalently be 
represented by the parallel combination of its conductance and capacitance, $G_{a}$ and $C_{a}$, respectively [1]. Further, the oscillator's equivalent circuit is then formed by connecting both the models regarded to the RTD device and antenna, as shown in Fig. 2.

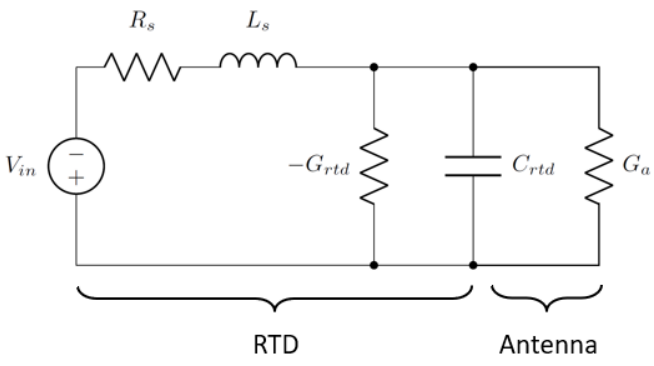

Figure 2. Equivalent circuit for the RTD-based oscillator.

In addition, it is necessary to explicitly the proposed formalism to evaluate the oscillating characteristic of such a circuit. The formalism incorporates fitting parameters extracted from the experimental current versus voltage characteristics (I-V) to further asses the width of the depletion region of the device. A straightforward expression that gives an excellent fit to the experimental data is provided by [7].

Further, it was observed that the influence of the large input voltages within the NDR leads for depletion's region profile similar to conventional diodes. Nonetheless, the hereproposed formalism introduces a fitting factor in order to express the width of the depletion region of RTDs adequately. Then, once considering that the characterization of the RTD device is known, the width of the depletion region is given by

$$
L_{d}=\left[\frac{2 \varepsilon_{d} V_{i n}}{q}\left(\frac{1}{N_{a}}+\frac{1}{N_{d}}\right)\right]^{M}
$$

where $\varepsilon_{d}$ is permittivity, $q$ is the electron charge, the $N_{a}$ and $N_{d}$ are the acceptor and donor concentrations in the collector region, respectively, $V_{\text {in }}$ is the input voltage and $M$ is an empiric factor estimated from the characterization of the devices. Full details on the proposed mathematical framework are available in [5].

After establishing the oscillating $\mathrm{THz}$ wave, the next step is to guide the $\mathrm{THz}$ beam radiated from the antenna of the oscillator circuit through the hemispherical lens. In addition, its behavior can be described by following S-parameters equations [1]

$$
\left(\begin{array}{ll}
S_{11} & S_{12} \\
S_{21} & S_{22}
\end{array}\right)=\left(\begin{array}{cr}
\Gamma_{s} e^{\mathrm{j} \Phi_{S}} & \eta_{s} \sqrt{\left(1-\Gamma_{s}^{2}\right)} e^{\mathrm{j} \Phi_{S i}} \\
\eta_{s} \sqrt{\left(1-\Gamma_{s}^{2}\right)} e^{\mathrm{j} \Phi_{S i}} & \Gamma_{s} e^{\mathrm{j} \Phi_{s}}
\end{array}\right)
$$

where $\eta_{s}$ denotes the power coupling efficiency from RTDbased oscillator to the free space, $\Gamma_{s}$ and $\Phi_{s}$ are the magnitude and phase of the $\mathrm{THz}$ signal reflectivity in the $\mathrm{Si}$ lens, respectively, and $\Phi_{S i}$ is the phase shift in the Si lens.

Further, the link is modeled as a transmission line, which is adapted from [1] to incorporates the phase shift and attenuation of the $\mathrm{THz}$ signal through the free space. In this case, the S-parameters are given by

$$
\left(\begin{array}{ll}
S_{11} & S_{12} \\
S_{21} & S_{22}
\end{array}\right)=\left(\begin{array}{rr}
0 & \alpha \\
\alpha & 0
\end{array}\right) e^{\frac{2 \pi L}{\lambda}}
$$

where $\alpha$ is the attenuation factor, $L$ is the length of the link and $\lambda$ is the wavelength of the THz signal.

Once all pertinent signal degradation effects have been accounted for the $\mathrm{THz}$ signal, the receiver modeling can be presented. Hence, the power coupling efficiency and the phase shift inside the antenna at the receiver side is accounted for the S-parameters given as [1]

$$
\left(\begin{array}{ll}
S_{11} & S_{12} \\
S_{21} & S_{22}
\end{array}\right)=\left(\begin{array}{lr}
\Gamma_{L} e^{\mathrm{j} \Phi_{L}} & \eta_{L} \sqrt{\left(1-\Gamma_{L}^{2}\right)} e^{\mathrm{j} \Phi_{a}} \\
\eta_{L} \sqrt{\left(1-\Gamma_{L}^{2}\right)} e^{\mathrm{j} \Phi_{a}} & 0
\end{array}\right)
$$

where $\eta_{L}$ is the power coupling efficiency at the receive antenna, $\Gamma_{L}$ and $\Phi_{L}$ are the magnitude and phase of the signal reflectivity, respectively, and $\Phi_{a}$ is the phase shift inside the receive antenna.

Finally, the overall system performance will be evaluated considering the ordinary On-Off keying (OOK) for the modulation data, which allow using directly of the expression provided in [1].

\section{RESULTS}

The mathematical formalism developed in the previous section is now applied to the $\mathrm{THz}$ proximity wireless communication system illustrated in Fig. 1. Before proceeding with the network performance analysis itself, it is interesting to analyse the signal evolution from generation until detection.

At this point, it is worth mentioning that the RTD device reported by Encomenderdo et al. [2] is utilized here only for convenience sake. Such device incorporates a permittivity $\varepsilon_{\text {GaN }}=83.67 \mathrm{pF} / \mathrm{m}$, a donor concentration in the collector region $N_{d}=10^{17} \mathrm{~cm}^{-3}$ and width of the depletion region $L_{d}=36 \mathrm{~nm}$ for a input voltage applied of $V=9.7 \mathrm{~V}$. The acceptor concentration value, on the other hand, was not available in the mentioned work and had to be inferred. To circumvent this issue, it was considered the empirical observation that the acceptor concentration value is approximately two orders of magnitude lower than the donor concentration in conventional diode junctions, and the value $N_{a}=1.47 \times 10^{15} \mathrm{~cm}^{-3}$ was adopted. Once the intrinsic parameters of the RTD device are known, it was allowed to estimate the fitting parameter $M=0.6673$ through (1). In possession of these variables and choosing $V_{\text {in }}=0.625 \mathrm{~V}$ and $R_{s}=1 \Omega$, additional calculations result in the following parameters to provide the oscillation: $L_{s}=10.95 \mathrm{pH}, C_{r t d}=$ 18.45 $\mathrm{fF}$ and $G_{r t d}=-20.64 \mathrm{mS}$. The idea is to check how the width of the depletion region and, consequently, the negative differential region (NRD) of the I-V characteristics depends on such a fitting parameter and distinct values on the input voltage. It is important to assert that this proposed approach shows a close agreement to the several device characterizations presented in the literature and the interested readers should refer to [5] for further details.

Now, the overall description of the transmitter can be achieved dealing with the effect of the transmitting antenna's coupling into the RTD device. In order to radiate the $\mathrm{THz}$ beam generated from the RTD, it was chosen bowtie antenna was selected because it provides a broader frequency 
bandwidth than, e.g., a dipole antenna [3]. Such a bowtie antenna has a $40 \Omega$ purely resistive impedance at the resonance frequency of $300 \mathrm{GHz}$ [3]. Although this value still is capable of maintaining the oscillation stable in the RTD device, it is too small to achieve a maximum power transference to the antenna. Thus, a step-up impedance transformer based on a transmission line was used to provide a load resistance $R_{L}=97 \Omega$ [3].

After, Table 1 shows all the relevant calculated parameters related to S-matrices related to hemispherical Si lens, link and receiver antenna.

\section{TABLE I. SYSTEM PARAMETERS}

\begin{tabular}{l|c|c}
\hline \multicolumn{1}{c|}{ Parameter } & Symbol & Value \\
\hline Magnitude of the signal reflectivity in the Si lens & $\Gamma_{S}$ & 0.3 \\
\hline Phase of the signal reflectivity in the Si lens & $\Phi_{S}$ & $\pi$ \\
\hline Phase shift in the Si lens & $\Phi_{S i}$ & $1.45 \times \pi$ \\
\hline Attenuation factor & $\alpha$ & $10 \mathrm{~dB} / \mathrm{km}$ \\
\hline Length of the link & $L$ & $100 \mathrm{~m}$ \\
\hline Wavelength of the signal & $\lambda$ & $0.98 \mathrm{~mm}$ \\
\hline $\begin{array}{l}\text { Power coupling efficiency at the receive antenna } \\
\text { receive antenna }\end{array}$ & $\eta_{L}$ & 0.4 \\
\hline $\begin{array}{l}\text { Phase of the signal reflectivity inside the receive } \\
\text { antenna }\end{array}$ & $\Gamma_{L}$ & 0.25 \\
\hline Phase shift inside the receive antenna & $\Phi_{L}$ & $\pi$ \\
\hline
\end{tabular}

With all appropriate transmitter's variables adequately described, the specificities related to the generated waveform can now be addressed. In the analyzed scenario, the RTDbased oscillator operates in the frequency of the order of 304.9 $\mathrm{GHz}$ with a peak voltage of $0.9308 \mathrm{~V}$ at the transmitter side. Initially, the absolute value of the generated wave voltage amplitudes (black curve) superposed by the wave voltage amplitudes (blue curve) that reach the receptor after the propagation of the $100-\mathrm{m}$-long $\mathrm{THz}$ link as a function of the time is shown in Fig. 3. As one might expect, the optical signal is continuously attenuated as it propagates through the optical fiber.

Next, it is investigated the bit error rate (BER) as a function of the received power in a system based on On-Off Keying (OOK) modulation, as shown in Fig. 4. The idea is to check how the attenuation influences the decision variable based on the signal-to-noise ratio (SNR) at the receiver side, particularly at distances that are compatible with the shortdistance $\mathrm{THz}$ communications (up to $100 \mathrm{~m}$ ). It can be observed from these results that the performance is highly degraded when longer transmission distances are required.

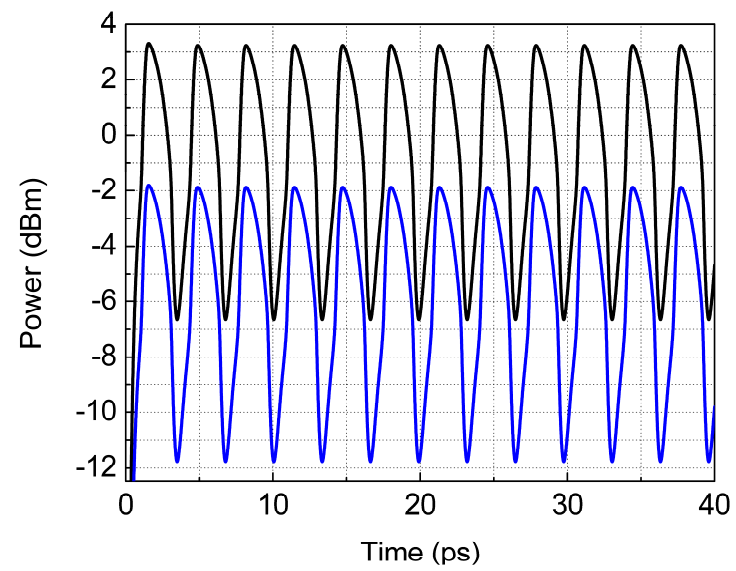

Figure 3. Time representation of the generated (black curve) and received (blue curve) THz signal after the propagation of the 100-mlong link.

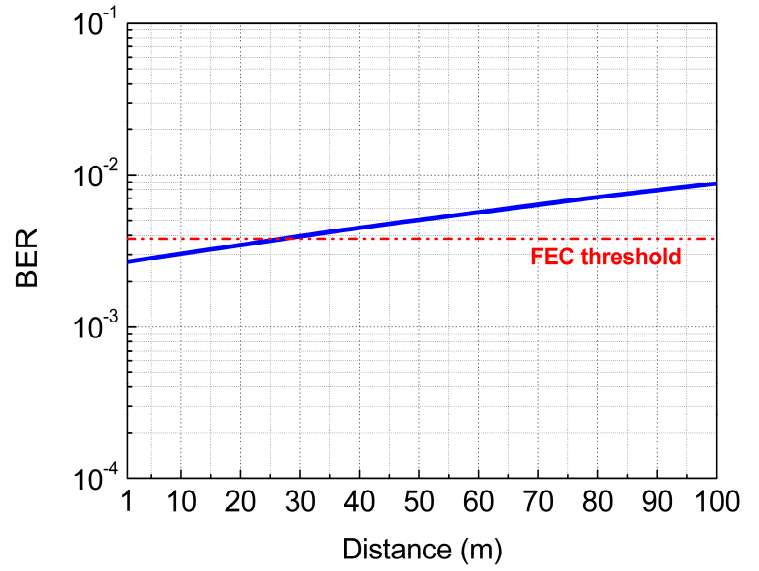

Figure 4. BER versus transmission distance for the $\mathrm{THz}$ communication system.

Nonetheless, this problem can be adequately circumvented with the adoption of a forward error correction (FEC) algorithms. The high BER observed for longer distances occurs since the FEC decoder becomes very inefficient when submitted to high BER transmissions at its input. The BER threshold by which the FEC decoder offers a substantial correction (close to the error-free zone) is approximately $3.8 \times 10^{-3}$ [4], which was able to reduce the BER down to acceptable levels up to $27 \mathrm{~m}$.

\section{CONCLUSION}

In this paper, it is have carried out a comprehensive analysis of a $\mathrm{THz}$ proximity wireless link that has an RTD device as a solid-state signal source. On top of that, was investigated how the RTD-based oscillator performs under DC bias applied within the NDR to provide oscillations in the $\mathrm{THz}$ range. The formalism accounted for the DC bias effect over the capacitance and inductance of the reactive elements that composed the small signal model of the RTD device. The results indicated that the RTD-based oscillator could stabilize very high-frequency oscillations and the $\mathrm{THz}$ proximity link considered is able to perform error-free transmissions at propagation distances up to $27 \mathrm{~m}$.

\section{REFERENCES}

[1] L.D. Manh et al., "External Feedback Effect in Terahertz Resonant Tunneling Diode Oscillators," in IEEE Transactions on Terahertz Science and Technology, vol. 8, no. 4, pp. 455-464, July 2018.

[2] J. Encomendero et al.: Room temperature microwave oscillations in GaN/AIN resonant tunneling diodes with peak current densities up to 220 kA/cm², Applied Physics Letters, vol. 112, 103101, Mar. 2018.

[3] Diebold, S., Nakai, S., Nishio, K., Kim, J.-Y., Tsuruda, K., Mukai, T., Fujita, M., and Nagatsuma, T.: 'Modeling and simulation of terahertz resonant tunneling diode based circuits', IEEE Trans. THz Sci. Technol., 2016, 6, (5), pp. 716-723.

[4] S. Jia, X. Yu, H. Hu, J. Yu, T. Morioka, P. U. Jepsen, and L. K. Oxenløwe, "120 Gb/s multi-channel $\mathrm{THz}$ wireless transmission and THz receiver performance analysis," IEEE Photon. Technol. Lett., vol. 29, no. 3, pp. 310-313, Feb. 2017.

[5] Nobrega. R.T, Duarte. U.R, Raddo. T.R, Glesk. I, Sanches. A.L, and Loiola. M.B, "A Semi-Analytical Approach for Performance Evaluation of RTD-based Oscillators," in 21th Int. Conf. Transparent Optical Networks, Angers, France, In Press, 2019.

[6] J. M. Gering, D. A. Crim, D. G. Morgan, P. D. Coleman, W. Kopp, and H. Morkoç, "A small-signal equivalent-circuit model for GaAsAlxGa1-x As resonant tunneling heterostructures at microwave frequencies," J. Appl. Phys., vol. 61, no. 1, pp. 271-276, Jan. 1987.

[7] J.N. Schulman, H.J. De Los Santos and D.H. Chow: Physics-based RTD current-voltage equation, IEEE Electron Device Letters, vol. 17, no. 5 , pp. 220-222, May 1996. 